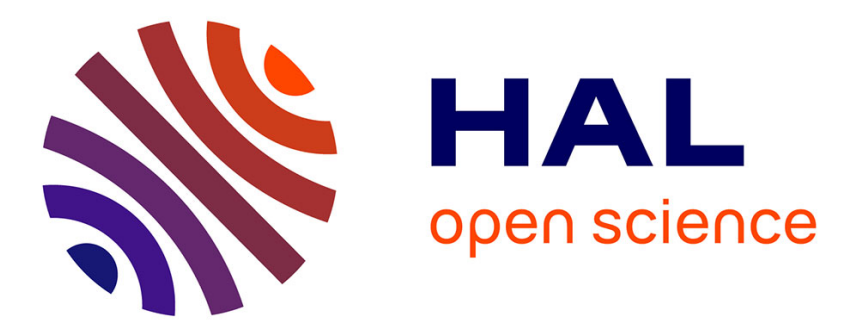

\title{
Fibrillogenesis from nanosurfaces: multiphoton imaging and stereological analysis of collagen 3D self-assembly dynamics
}

\author{
Stéphane Bancelin, Etienne Decencière, Vaïa Machairas, Claire Albert, \\ Thibaud Coradin, Marie-Claire Schanne-Klein, Carole Aimé
}

\section{To cite this version:}

Stéphane Bancelin, Etienne Decencière, Vaïa Machairas, Claire Albert, Thibaud Coradin, et al.. Fibrillogenesis from nanosurfaces: multiphoton imaging and stereological analysis of collagen 3D selfassembly dynamics. Soft Matter, 2014, pp.6651-6657. 10.1039/c4sm00819g . hal-01082854

\section{HAL Id: hal-01082854}

https://hal-mines-paristech.archives-ouvertes.fr/hal-01082854

Submitted on 9 Jan 2015

HAL is a multi-disciplinary open access archive for the deposit and dissemination of scientific research documents, whether they are published or not. The documents may come from teaching and research institutions in France or abroad, or from public or private research centers.
L'archive ouverte pluridisciplinaire HAL, est destinée au dépôt et à la diffusion de documents scientifiques de niveau recherche, publiés ou non, émanant des établissements d'enseignement et de recherche français ou étrangers, des laboratoires publics ou privés. 


\title{
Fibrillogenesis from Nanosurfaces: Multiphoton Imaging and Stereo- logical Analysis of Collagen 3D Self-assembly Dynamics ${ }^{\dagger}$
}

\author{
Stéphane Bancelin, ${ }^{a}$ Etienne Decencière, ${ }^{* b}$ Vaïa Machairas, ${ }^{b}$ Claire Albert, ${ }^{c}$ Thibaud Coradin, ${ }^{c}$ \\ Marie-Claire Schanne-Klein, ${ }^{* a}$ and Carole Aimé ${ }^{* c}$
}

\author{
Received Xth $X X X X X X X X X X 20 X X$, Accepted $X$ th $X X X X X X X X X 20 X X$ \\ First published on the web $X$ th $X X X X X X X X X X 200 X$ \\ DOI: 10.1039/b000000x
}

The assembly of proteins into fibrillar structures is an important process that concerns different biological contexts, including molecular medicine and functional biomaterials. Engineering of hybrid biomaterials can advantageously provide synergetic interactions of the biopolymers with an inorganic component to ensure specific supramolecular organization and dynamics. To this aim, we designed hybrid systems associating collagen and surface-functionalized silica particles and we built a new strategy to investigate fibrillogenesis processes in such multicomponents systems, working at the crossroads of chemistry, physics and mathematics. The self-assembly process was investigated by bimodal multiphoton imaging coupling second harmonic generation (SHG) and 2 photons excited fluorescence (2PEF). The in-depth spatial characterization of the system was further achieved using the three-dimensional analysis of the SHG/2PEF data via mathematical morphology processing. Quantitation of collagen distribution around particles offers strong evidence that the chemically-induced confinement of the protein on the silica nanosurfaces has a key influence on the spatial extension of fibrillogenesis. This new approach is unique in the information it can provide on 3D dynamic hybrid systems and may be extended to other associations of fibrillar molecules with optically-responsive nano-objects.

\section{Introduction}

Controlling the association of nanomaterials with selfassembling biomolecules is a topic of high relevance in many fields, from therapeutics to biomaterials engineering. Protein fibrils received specific attention due to their implication in neurodegenerative disorders such as Alzheimer's and Parkinson's diseases ${ }^{1,2}$. In this context, self-assembling proteins and peptides, such as human serum albumin ${ }^{3}$, islet- ${ }^{4}$ and beta-amyloid polypeptide ${ }^{5-10}$, and human beta2-microglobulin ${ }^{11}$ have been associated with inorganic particles to investigate fibrillogenesis processes and kinetics. However the outcomes of these works are often divergent. On the one hand, particles could promote aggregation by a condensation-ordering mechanism induced by an increase of the local concentration of peptides in the vicinity of

† Electronic Supplementary Information (ESI) available: SHG photon density around particles (at 4 sequential times, for 5 samples involving NP-SiSO3 and 5 NP-Si samples); Quantitation of collagen fibrillogenesis using a statistical approach. See DOI: 10.1039/b000000x/

a Laboratoire d'Optique et Biosciences, CNRS ; Ecole Polytechnique ; Inserm U696, 91128 Palaiseau, France. E-mail: marie-claire.schanneklein@polytechnique.edu

${ }^{b}$ Centre de Morphologie Mathematique, MINES ParisTech, Fontainebleau, France.E-mail: etienne.decenciere@mines-paristech.fr

${ }^{c}$ Sorbonne Universités, UPMC Univ Paris 06, CNRS, Collège de France, UMR 7574, Chimie de la Matière Condensée de Paris, F-75005, Paris, France. E-mail: carole.aime@upmc.fr the nanoparticles ${ }^{3,5,11}$. On the other hand, particles have also been shown to slow down fibrillogenesis by depleting the amount of free monomers ${ }^{4,6,8,9}$. Meanwhile, it comes up that particles size, chemistry, and concentration play a crucial role in those assembly processes ${ }^{7,10}$. Such studies are also of primary importance in current nanoscience since the combination of self-assembling macromolecules with nanoscale objects has emerged as a key strategy to build bio-functional systems ${ }^{12,13}$. It is therefore necessary to develop new strategies to investigate biological self-assembly processes at inorganic interfaces at the supramolecular level for their use in nanomedicine and nanotechnology.

Type I collagen is a typical example of biomolecule whose controlled self-assembly at the interface with mineral phases is of major concern when designing biomedical materials ${ }^{14-16}$. Type I collagen is the main structural protein of connective tissues, giving form and strength to the body. Synthesized by cells as triple helix units, collagen self-assembles into fibrils in vivo and in vitro, forming three-dimensional (3D) matrices $^{17-19}$. The effect of silica in different forms, from silicic acid to colloidal silica, on Type I collagen self-assembly has been previously reported, demonstrating that non-specific interactions could result either in fibrillogenesis enhancement or inhibition ${ }^{20}$. More recently we showed that a suitable surface modification of silica nanoparticles allows the formation of 
well-defined collagen triple helices-coated colloids that preserve the fibrillogenesis properties of the protein ${ }^{21}$. However the detailed analysis of the growth and 3D organization of such multi-scale dynamic bio-mineral networks faces important conceptual and technical challenges. In particular, a simultaneous monitoring of both the particle and the protein has to be achieved.

Up to now, time-lapse confocal optical microscopy is the most commonly used approach to follow protein self-assembly, although it requires complementary protein tagging with fluorescent molecules or antibodies ${ }^{22,23}$. As an alternative, it has been demonstrated that multiphoton microscopy taking advantage of intrinsic Second Harmonic Generation (SHG) signal from fibrillar collagen and combined with specific image processing provides access to new quantitative measurements on collagen-based biological tissues and biomaterials in two dimensions $^{24-30}$ and, more recently, in three dimensions ${ }^{31,32}$. On this basis, we propose here a powerful tool to study collagen fibrillogenesis in 3D composites, by applying recent developments in stereological analysis to a combination of SHG images of the collagen fibrils with Two-Photon Excited Fluorescence (2PEF) images of the fluorescently-labeled silica particles. It thus becomes possible to monitor the course of the fibrillogenesis process from the nanoparticle surface without any protein labeling thanks to the high specificity of SHG microscopy to collagen fibrils. This approach is particularly useful to get a comprehensive view of the influence of particle surface chemistry on the self-assembly of hybrid systems. As this novel methodology may be further applied to a large diversity of fibrillar biological macromolecules (e.g. collagen, myosin, tubulin) ${ }^{33}$ and fluorescent nanoparticles (Fig.S1), it constitutes a key tool for the design and investigation of biofunctional materials from engineered nano-surfaces.

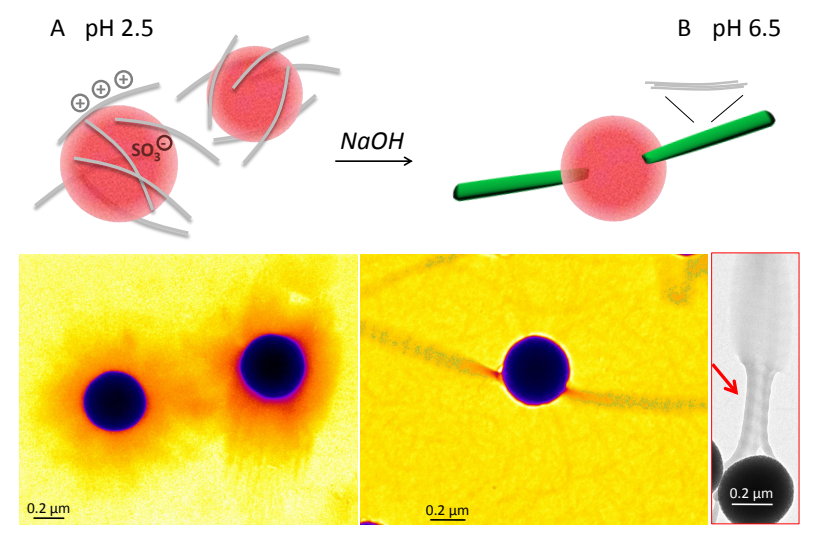

Fig. 1 Collagen (A) adsorption and (B) fibrillogenesis from $\mathrm{NP}_{-} \mathrm{SiSO}_{3}$ nano-surfaces, as shown by Transmission Electron Microscopy.

\section{Three approaches, one system}

\subsection{Engineering silica-collagen biocomposites}

Type I collagen is soluble in acidic medium, where it is stabilized as individual triple helices $(300 \mathrm{~nm}$ in length, 1.5 $\mathrm{nm}$ in diameter). Near neutral $\mathrm{pH}$ conditions, collagen selfassembles forming fibrils ca. $100 \mathrm{~nm}$ in diameter and up to 1 $\mathrm{mm}$ in length ${ }^{17,18}$. This $\mathrm{pH}$-dependent self-assembling property can be used to drive the formation of synthetic networks made up of silica particles and collagen. A pre-requisite to obtain such extended hybrid network via a bottom-up approach is to design well-defined collagen-silica starting units (Fig.1A). From the protein point of view, acidic conditions (acetic acid solution, $\mathrm{pH} 2.5$ ) are required to ensure the stabilization of collagen triple helices ${ }^{21}$. Considering the nanoparticle design, it is important to rationally define its dimension and surface chemistry, which are key parameters for controlling protein adsorption at the molecular level ${ }^{34-36}$. On this basis, we have used silica particles (NP-Si) having a diameter of $290 \mathrm{~nm}$, thus matching the largest dimension of collagen triple helices. Furthermore, surface modification with negatively-charged sulfonate groups (NP-SiSO ${ }_{3}$ ) was performed to control its interaction with positively-charged collagen via electrostatic interactions. We recently demonstrated that this approach allows significant binding of collagen triple helices on the particle, providing a good platform to trigger fibrillogenesis from the nano-surface upon increasing $\mathrm{pH}(\text { Fig. } 1 B)^{21}$. However, a deeper understanding of the influence of the nano-surface on fibrillogenesis requires the development of new approaches to investigate the dynamics of the self-assembly process, as well as the supramolecular organization and morphology of collagen fibrils. In particular, a simultaneous monitoring of both the particles and the proteins has to be achieved.

\subsection{Combined SHG/2PEF imaging}

Here we combined SHG imaging to follow the real-time growth of unlabelled collagen fibrils with $2 \mathrm{PEF}$ imaging of $\mathrm{NP}-\mathrm{Si}$ and $\mathrm{NP}-\mathrm{SiSO}_{3}$ after incorporation of suitable fluorophores (Alexa Fluor $\AA$ 488) within the particles core (see Fig.2A). To ensure significant reproducibility, 5 samples for each particle chemistry were investigated (5 $\mathrm{NP}-\mathrm{SiSO}_{3}$ and 5 NP-Si samples, Fig.S2). 3D rendering of bimodal image stacks reveals the onset of fibrils growth around the 3D distribution of fluorescent nanoparticles (Fig.2B). The fluorescence signal (Fig.2C) may in fact correspond either to single NP$\mathrm{SiSO}_{3}$ or NP-Si particles or to clusters of such particles, and both objects will be designated as NPs hereafter. SHG reveals fibrillar structures with diameters limited by the optical resolution (Fig.2D), which are mostly single collagen fibrils but may also correspond to fibrils assemblies, while the centrosymmetrical liquid solutions exhibit no signal ${ }^{37}$. This structural 
specificity of SHG for aligned collagen assemblies is related to the physics of this coherent multiphoton signal ${ }^{38-40}$. At the molecular scale, SHG signals correspond to strong electronic polarization of the peptide bonds that radiate at the harmonic frequency due to their noncentrosymmetrical environment. The SHG signal of macro-molecular collagen distributions then corresponds to the summation of all these electric field radiations, which efficiently amplifies SHG in dense and aligned materials such as collagen fibrils. In contrast, SHG vanishes for centrosymmetrical distributions such as collagen solutions, and SHG microscopy is therefore a structural probe of collagen fibril formation.

Altogether, the SHG signal intensity scales quadratically with the number of aligned collagen triple helices. Accordingly, increasing SHG signals indicate increasing fibril diameter or packing. Basic quantitation of the collagen network may then be obtained by measuring the total SHG signal or the number of voxels with significant SHG signal in the image ${ }^{28}$. This method was used recently to monitor collagen fibrillogenesis in various physico-chemical conditions ${ }^{41}$. However, a new approach is required here to quantify the collagen $3 \mathrm{D}$ assembly around the NPs.

\subsection{D analysis of SHG/2PEF data}

Precise quantitation of collagen fibrillogenesis around NPs is difficult. A manual approach is not only tedious and timeconsuming but, more importantly, insufficiently repeatable and observer-dependent. In addition, a two-dimensional analysis of the presented model would be clearly inappropriate, as one can confidently hypothesize that the influence of nanoparticles on collagen fibrillogenesis is three-dimensional. Therefore, an automatic 3D image processing approach was deemed compulsory.

A model, based on mathematical morphology ${ }^{42,43}$, is introduced here to precisely define nanoparticles and collagen fibrils in our images and allow for their quantitation. Note that no image registration method is used because the collagensilica network may move and deform in the liquid solution during the fibrillogenesis process. We rather introduce two statistic measures in order to spatially characterize the effect of nanoparticles on collagen fibrillogenesis. First, we measure fibrillar collagen density with respect to the distance to nanoparticles. Second, we count the number of collagen fibrils around nanoparticles, at a given distance range.

The main processing steps are illustrated in Fig. $2 E-H$, and described in the following section.

\section{Three-dimensional analysis}

\subsection{Segmentation and labelling of NPs}

The first issue is to segment the nanoparticles, which later enables to separate each NP and perform an individual analysis for fibril counting. This segmentation (see Methods) results in a binary image. One can notice that nanoparticles are in fact represented by groups of close but non-adjacent connected components. In order to separate them without introducing over-segmentation, we use a generalized connectivity, as introduced by Serra ${ }^{44}$ : we consider that two voxels are neighbours when they are separated by less than $2 \mu \mathrm{m}$. Using this connectivity we associate a unique label $i(1 \leq i \leq N)$ to each nanoparticle. Hereafter, $P_{i}$ will denote the nanoparticle labelled with $i$. The influence zone $Z_{i}$ of the particle $P_{i}$ is then given by the set of pixels which are closer to $P_{i}$ than to any other particle $P_{j}$ or the image border (Fig.2E).

In order to quantify the spatial influence of nanoparticles on collagen fibrillogenesis, a distance function is computed around them: for each background voxel $x$, its distance $d(x)$ (in $\mu \mathrm{m}$ ) to the surface of the closest nanoparticle is computed. As we want to avoid possible biases introduced by the image borders, if the distance of a background voxel $x$ to the image border is smaller than $d(x)$, then $d(x)$ is set equal to $\infty$, which in practice means that this voxel is not taken into account in the following steps. This precaution is necessary, as nanoparticles outside the microscope field of view can also influence fibrillogenesis. A quantized distance $c$ is finally computed with factor $q$ : $c(x)=\lceil d(x) / q\rceil$, where $\lceil y\rceil$ denotes the smallest integer larger than the real number $y$. The set $C_{j}$ corresponds to the voxels $x$ such that $c(x)=j$. We take $q=1 \mu m$ which is approximately the axial resolution of the multiphoton microscope (Fig.2F).

\subsection{Fibrillar collagen density around NPs}

For each distance class $C_{j}$, the mean value of the SHG signal among all voxels belonging to this image class was computed:

$$
\rho\left(C_{j}\right)=\frac{\sum_{x \in C_{j}} \operatorname{shg}(x)}{\sum_{x \in C_{j}} 1} .
$$

The result is given in photon number and is correlated to the squared density of fibrillar collagen. The computation of $\rho\left(C_{j}\right)$ shows that SHG photon density increases with time, being always maximal close to the particle surface and exponentially decaying with increasing distance (Fig. $3 A$ ). This surface-mediated fibril growth was found to be highly reproducible, while with bare nanoparticles non-monotonic variations with respect to the particle surface were observed (Fig.S2). This indicates that there is no systematic correlation 


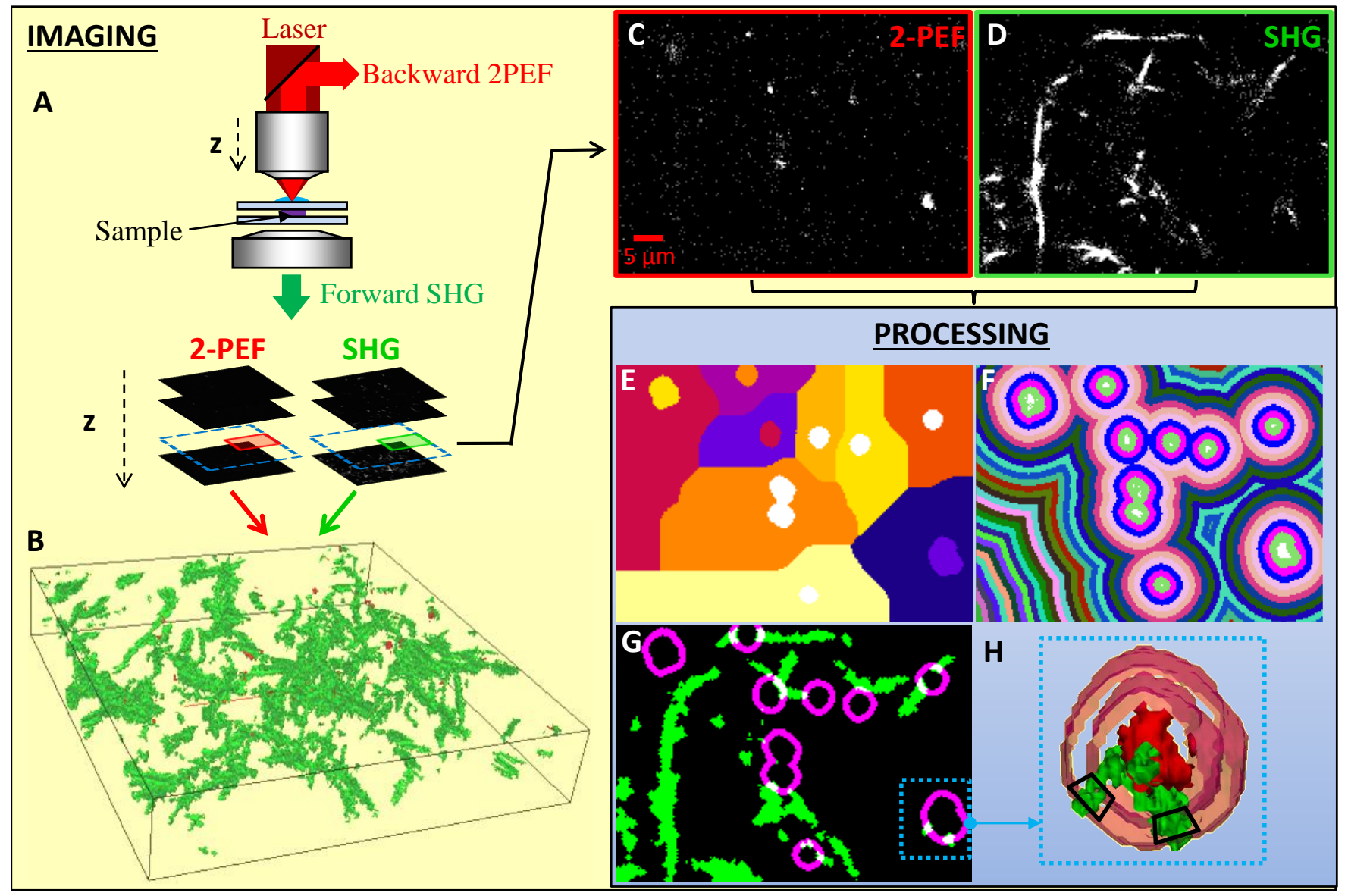

Fig. 2 3D analysis of collagen - NPs composites. (A) Multiphoton setup, with simultaneous forward detection of SHG signals and backward detection of 2PEF signals, providing multimodal image stacks; Multiphoton data: (B) 3D rendering of the sample: red structures correspond to the 2 PEF image of NPs, and green ones to the SHG image of collagen fibrils. Field of view: 154 x $160 \times 30 \mu m^{3}$. A zoomed-in region of (B) can be seen on images (C) and (D), respectively for 2PEF and SHG channels, on which image processing is thereafter illustrated: (E) NPs influence zones $Z_{i}$; (F) Distance classes $C_{i}$ around NPs; (G) Distance class $C_{2}$ on pre-processed collagen fibrils image; (H) 3D rendering of a zoomed-in region of $(\mathrm{G})$ containing one NP : intersections between $C_{2}$ and two collagen fibrils. 
between the presence of non-functionnalized NP-Si surfaces and the course of fibrillogenesis.
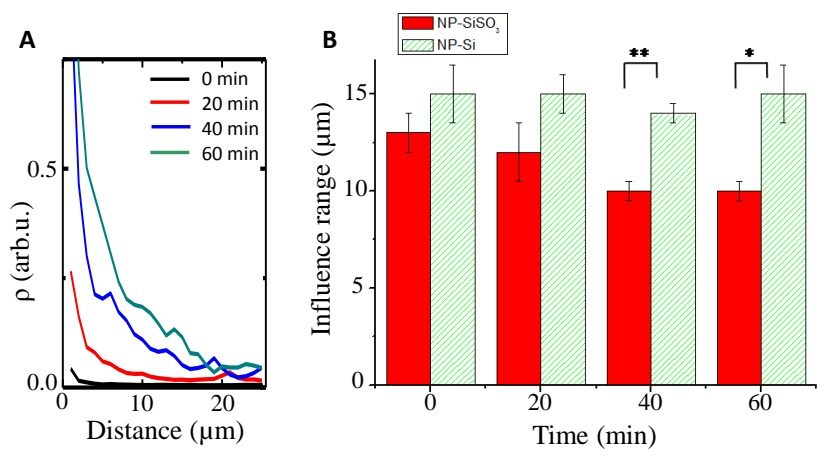

Fig. 3 (A) SHG photon density around NP-SiSO 3 particles imaged every 20 minutes during an hour to study the onset of collagen fibrillogenesis. (B) Influence range for NP-Si (green bars) and $\mathrm{NP}-\mathrm{SiSO}_{3}$ (red bars). The values are the average influence range over five samples at every time. The error bars indicate the standard error of the mean.

In order to quantify collagen fibrillogenesis around NP-Si and $\mathrm{NP}-\mathrm{SiSO}_{3}$ particles, we defined the NP influence range as the distance $d$ splitting the SHG signal integral in two equal parts:

$$
\sum_{j \leqslant d} \sum_{x \in C_{j}} \operatorname{shg}(x)=\frac{1}{2} \sum_{j<\infty} \sum_{x \in C_{j}} \operatorname{shg}(x) .
$$

The time evolution of the averaged influence range for both types of samples are displayed in Fig.3C, where the error bars represent the standard error of the mean (SEM) over the five independent samples.

Initially, at $t=0$, as there is almost no collagen fibrils in the samples, the influence range is dominated by the SHG background over the field of view, and is basically equal to a quarter of the mean distance between two nanoparticles. Thereby, as expected, the mean influence range of the two populations of samples are the same. Then, in $\mathrm{NP}_{-} \mathrm{SiSO}_{3}$ samples, the influence range decreases with time. This suggests a compaction effect, where the fibrillogenesis process taking place at the vicinity of the particle surface occurs at the detriment of fibrils elongation in the bulk solution. Interestingly, the influence range tends to level off after 40 minutes, i.e. considering SHG data, the system does not evolve anymore after this delay. Kinetics investigations based on a global SHG signal quantitation as previously reported ${ }^{41}$ provide an exponential rising time of $13 \pm 3 \mathrm{~min}$ for fibrillogenesis in presence of NP-SiSO 3 (Fig.S3). This value is much smaller than $54 \pm$ 14 min previously obtained in similar conditions in absence of particles ${ }^{41}$, and suggests the occurence of a condensationordering mechanism responsible for fastening fibrillogenesis.
In sharp contrast, for NP-Si samples, the influence range remains constant over time (Fig. $3 \mathrm{C}$ ), confirming that fibrillogenesis does not occur systematically from the surface, but also independently of the surface. In this case, fibrillogenesis kinetics is characterized by an exponential rising time of $30 \pm 8$ min, which corresponds to an intermediate value, in between $\mathrm{NP}-\mathrm{SiSO}_{3}$ and the absence of particles. This indicates that bare silica nanosurface can influence fibrillogenesis via some weak adsorption of triple helices and/or fibrils but that these interactions cannot specifically direct collagen self-assembly. In addition, after 60 minutes, where the influence range values are stable for both sets of samples, associated standard deviation is markedly smaller for NP-SiSO ${ }_{3}$ compared to NP$\mathrm{Si}$. Altogether, these data demonstrate that sulfonated surfaces provide a significant and reproducible control on the fibrillogenesis process over the whole experimental timescale.

\subsection{Collagen 3D structure around NPs}

To compute the number of collagen fibrils around NPs, it was again necessary to identify only the collagen fibrils after segmentation. This requires to extract the SHG photons at the interface that can not be attributed to a given fibril and that results from the accumulation of aligned collagen molecules or short fibrils at the particle surface with sizes and interdistances smaller than the optical resolution. To do so, we removed from the resulting mask all voxels, which belong to $C_{1}$ and the NPs. This implements our definition of a fibril, that should extend over more than $1 \mu \mathrm{m}$; i.e. over at least two distance classes. A small dilation was then applied to avoid SHG fibril splitting provoked by noise. Afterwards, we labeled the resulting binary image, obtaining labels $\left\{F_{j}\right\}$ for the resulting fibrils. This procedure is illustrated in Fig.2G.

Finally, for each nanoparticle $P_{i}$, we computed the number $n_{i}$ of collagen fibrils that intersect its influence zone $Z_{i}$ and distance class $C_{2}$ (Fig.2H):

$$
n_{i}=\operatorname{Card}\left\{F_{j} \mid F_{j} \cap Z_{i} \neq \emptyset, F_{j} \cap C_{2} \neq \emptyset\right\} .
$$

The results are presented in Fig. $4 A$ for a NP-SiSO ${ }_{3}$ sample. It clearly shows the formation of new collagen fibrils with time around NPs. Averaged results for all samples are displayed in Fig. $4 B, C$. It is interesting to note that the number of fibrils per NP ranges between 0.6 and 0.9 (Fig. $4 B$ ), while only ca. $50 \%$ of the NPs are associated with at least one fibril after $60 \mathrm{~min}$ utes (Fig.4C). Taken together, these data suggest that some particles bear a small number of discrete isolated fibrils that extend beyond the particle close vicinity, i.e. beyond the first distance class as illustrated in Fig. $2 \mathrm{H}$, while others most probably bear shorter fibrils. Indeed, the formation of short fibrils, hundreds nm in length, at the surface of $\mathrm{NP}-\mathrm{SiSO}_{3}$, has been previously ascertained by electron microscopy ${ }^{21}$. However, 

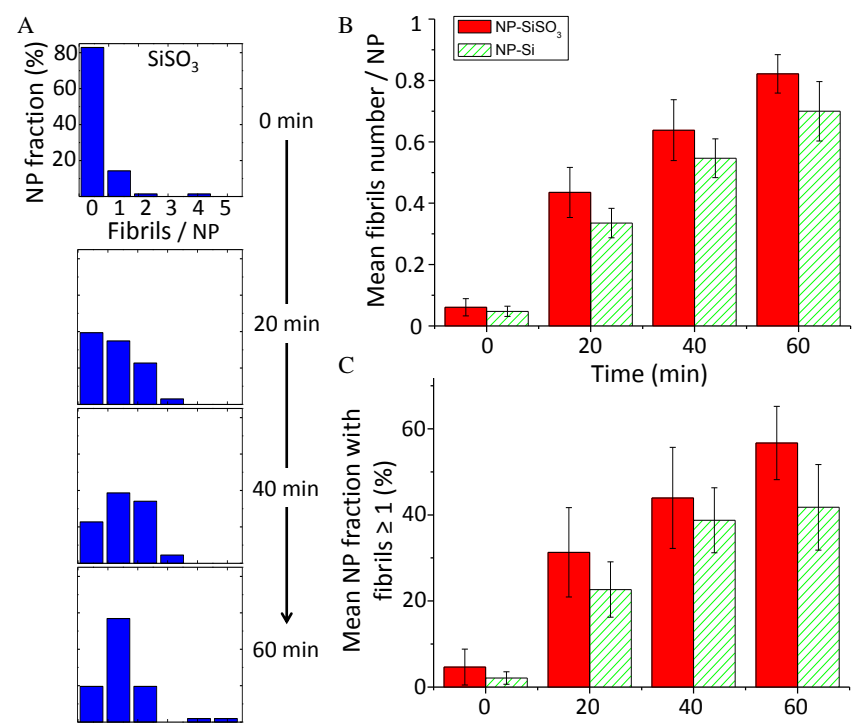

Fig. 4 (A) Fibrils number per particle at each time for a $\mathrm{NP}-\mathrm{SiSO}_{3}$ sample. (B) Mean fibrils number per particle and (C) mean proportion of particles with at least one fibril attached.

when coming to the comparison between NP-SiSO ${ }_{3}$ and NPSi samples, the computed values are statistically similar. This means that the number of individual fibrils that can be distinguished and associated to a given particle is virtually the same in both systems. Yet the shorter influence range previously measured for NP-SiSO 3 samples indicates that the SHG photon density is higher around functionalized NPs than around bare ones. This can correspond either to the formation of larger collagen fibrils or to a denser packing of fibrils near the $\mathrm{NP}-\mathrm{SiSO}_{3}$ surface, both of which would equally contribute to the enhancement of SHG intensity. Interestingly, this is reminiscent of rod-like dense collagen structures commonly observed at the surface of functionnalized particles, that exhibit a marked contrast under the electron beam (Fig.1B, arrow in insert).

\section{Discussion and Conclusions}

Investigating the dynamics of protein fibrillogenesis in 3D is a challenging task. Further introduction of nanoscale objects as a mean to direct the fibrillogenesis process raises more complex issues related to the simultaneous monitoring of the inorganic and bio-organic components as well as the quantitation of interfacial processes. Here a combination of two independent non interfering multiphoton imaging techniques is used to specifically monitor each partner during the self-assembly process, as well as to determine their relative spatial evolution. Mathematical morphology analysis is then applied to 3D images and, based on an appropriate stereological definition of the hybrid network and its interfaces, provides quantitative evidence of the influence of the inorganic nanoscale surface on the protein organization.

Our approach allowed the precise quantitation of collagen assembly with respect to the distance to the silica particle surface, leading us to define and determine the influence range of the particle. Importantly, diluted collagen provides suitable conditions to specifically focus on the growth of isolated fibrils. High collagen density around sulfonated silica particles together with decreasing influence range with time strongly suggest that fibrillogenesis proceeds from the surface of the particle. This can be attributed to an increase of the local collagen concentration at the interface due to the electrostatic interactions between the sulfonate groups and the collagen triple helices that promotes collagen ordering and fibrils growth. This hypothesis is supported by the faster kinetics of fibrillogenesis compared to collagen alone. Indeed, at $\mathrm{pH} 2.5$, sulfonated particles exhibit a high negative charge density allowing for an efficient pre-confinement of positively-charged triple helices. Since the pKa of the sulfonate function is 1.8, the particle charge density, and therefore its interactions with collagen, is almost constant during the $\mathrm{pH}$ increase triggering the self-assembly process. In contrast, the bare silica nanoparticles have a point of zero charge of ca. $3^{35}$, meaning that they initially have no favorable electrostatic interactions with triple helices and that their surface charge will significantly evolve during the course of fibrillogenesis. Accordingly, for bare silica nanoparticles, the influence range does not evolve with time and the system shows poor reproducibility, suggesting that fibrillogenesis does not occur systematically from the particle surface. Our data thus shows that the pre-confinement onto NP-SiSO 3 associated with accumulation of triple helices is a key step to promote collagen ordering and fibrils growth. One step further, it was possible to determine the number of collagen fibrils around individual particles. However, it was not possible to apply this procedure to the area at the close vicinity of the particle surface, where SHG photons cannot be attributed to a discret object with dimensions matching the optical resolution. This reflects that the spatial resolution of the mathematical discretization procedure is limited by the resolution of the imaging technique. This led us to remove the first distance class from the computed data. In these conditions, NP-Si and NP-SiSO ${ }_{3}$ systems became statistically similar, despite the fact that mean values of fibril numbers around nanoparticles are systematically higher for sulfonated silica surfaces. This would suggest that the first distance class is the area where the two systems are more markedly different, enlighting the key step of fibrillogenesis that occurs at functionalized interfaces.

Stereological discrimination of individual fibrils allows the correlation between the SHG photon density and the number of fibrils around particles. This ultimately provides informa- 
tion on collagen organization (i.e. supramolecular packing and fibrils morphology) down to the sub-micronic scale from an optical method. Especially, it underlines the role of sulfonated particles in the confinement and accumulation of triple helices that self-assemble from the surface, where the growth of fibrils in a given direction is fed by the desorption of neighbour proteins. In other words, the confined triple helices can be considered as a reservoir for fibrillogenesis; upon increasing $\mathrm{pH}$, the local ordering of triple helices leads to the formation of dense rod-like structures from which fibrillogenesis further proceeds (Fig.S4).

The present work demonstrates the strength of combining nanochemistry, bio-imaging and morphological analysis to elucidate self-assembly processes in multi-scale hybrid dynamic systems. In particular, thanks to the simultaneous monitoring of the inorganic and bio-organic components and to the quantitation of interfacial processes, it demonstrates that after suitable chemical modification, silica nanoparticles can act as well-defined nucleation surfaces to favor and orient collagen fibrillogenesis. Ultimately, the here-presented approach and foreseen developments may be easily applied to a broad diversity of systems combining optically responsive inorganic nano objects with fibrillar proteins to be used in nanomedicine and nanotechnology.

\section{Materials}

\section{Synthesis of silica particles}

Stöber silica particles were synthesized and functionalized as previously described ${ }^{21}$. Alexa Fluor $\mathbb{R}$ ) 488 (Molecular Probes, Invitrogen) was covalently grafted within NP-Si and $\mathrm{NP}-\mathrm{SiSO}_{3}$ to obtain fluorescent nanoparticles according to literature $^{45}$.

\section{Sample preparation}

Type I collagen was extracted and purified from rat tail tendons as previously described ${ }^{19}$. All other chemicals were purchased and used as received. Water was purified with a Direct-Q system (Millipore Co.). Collagen $(100 \mu \mathrm{g} / \mathrm{ml})$ was solubilized in $0.5 \mathrm{M}$ acetic acid and fibrillogenesis was triggered upon increasing $\mathrm{pH}$ to 7.5 by addition of $\mathrm{NaOH}$. A drop of sample was placed between two glass coverslips and directly visualized using SHG microscopy without any staining.

\section{Multiphoton imaging}

In situ SHG/2PEF images of silica-collagen hybrid biocomposites (5 NP-SiSO 3 and $5 \mathrm{NP}-\mathrm{Si}$ samples) were acquired for 1 hour every 20 minutes from the onset of $\mathrm{pH}$ triggered self-assembly. We used circularly-polarized $860 \mathrm{~nm}$ excitation and a high NA objective (40x 1.1NA LD C-Apochromat, Zeiss) to achieve a focal volume of typically $362 \mathrm{~nm}$ lateral and $1.11 \mu \mathrm{m}$ axial dimensions (full width at $1 / \mathrm{e}$ ). SHG signals were detected in the forward direction and $2 \mathrm{PEF}$ signals in the backward direction, using appropriate spectral filters. We recorded $160 \times 160 \times 30 \mu m^{3}$ 3D images with $0.27 \times$ $0.27 \times 0.5 \mu \mathrm{m}^{3}$ voxel size and $10 \mu \mathrm{s}$ dwell time, resulting in 5 minutes total acquisition time. Using $50 \mathrm{~mW}$ excitation power at focus, we observed a limited photobleaching of the stained NP, while SHG was not affected as expected for such a non-resonant signal.

\section{D analysis}

All processing was done in $3 \mathrm{D}$, using a cubic connectivity (the neighbors of a given voxel are its 26 closest voxels: 8 in the same slice, and 9 in each adjacent slice), except when otherwise specified. In order to segment the nanoparticles, a median filter of size 2 is applied to the 2PEF image, followed by a hysteresis thresholding. Then, all connected components which are not present in at least two consecutive slices in the image stack are removed. Indeed, given the size of the nanoparticles, and the acquisition conditions, each nanoparticle should span at least two slices. To segment the collagen fibrils, we first applied a median filter of size 2 to the SHG image, followed by a $3 \mathrm{D}$ reconstruction, and a threshold at level 1.

\section{Acknowledgments}

C. Aimé thanks Sandrine Quignard for her help in the synthesis of fluorescent nanoparticles, Gervaise Mosser for fruitful discussions and comments on this work, and the ANR-Retour post-doctorant for financial support (ANR-09-RPDOC-02301).

\section{References}

1 F. Chiti and C. M. Dobson, Annu Rev Biochem, 2006, 75, 333-366.

2 D. Kashchiev, J Chem Phys, 2013, 139, 105103.

3 C. H. Vannoy and R. M. Leblanc, J Phys Chem B, 2010, 114, 1088110888.

4 C. Cabaleiro-Lago, I. Lynch, K. A. Dawson and S. Linse, Langmuir, 2010, 26, 3453-3461.

5 W.-h. Wu, X. Sun, Y.-p. Yu, J. Hu, L. Zhao, Q. Liu, Y.-f. Zhao and Y.-m. Li, Biochem Biophys Res Commun, 2008, 373, 315-318.

6 C. Cabaleiro-Lago, F. Quinlan-Pluck, I. Lynch, S. Lindman, A. M. Minogue, E. Thulin, D. M. Walsh, K. A. Dawson and S. Linse, J Am Chem Soc, 2008, 130, 15437-15443.

7 C. Cabaleiro-Lago, F. Quinlan-Pluck, I. Lynch, K. A. Dawson and S. Linse, ACS Chem Neurosci, 2010, 1, 279-287. 
8 L. Xiao, D. Zhao, W.-H. Chan, M. M. Choi and H.-W. Li, Biomaterials, 2010, 31, 91-98.

9 S. I. Yoo, M. Yang, J. R. Brender, V. Subramanian, K. Sun, N. E. Joo, S.H. Jeong, A. Ramamoorthy and N. A. Kotov, Angew Chem Int Ed, 2011, 50, 5110-5115.

10 H.-M. Chan, L. Xiao, K.-M. Yeung, S.-L. Ho, D. Zhao, W.-H. Chan and H.-W. Li, Biomaterials, 2012, 33, 4443-4450.

11 S. Linse, C. Cabaleiro-Lago, W.-F. Xue, I. Lynch, S. Lindman, E. Thulin, S. E. Radford and K. A. Dawson, Proc Natl Acad Sci USA, 2007, 104, 8691-8696

12 E. Gazit, J Chem Phys, 2007, 36, 1263-1269.

13 T. P. J. Knowles and M. J. Buehler, Nature Nanotechnol, 2011, 6, 469479.

14 M.-M. Giraud-Guille, C. Hélary, S. Vigier and N. Nassif, Soft Matter, 2010, 6, 4963-4967.

15 C. Aimé and T. Coradin, J Polym Sci Part B, 2012, 50, 669-680.

16 S. Heinemann, T. Coradin and M. F. Desimone, Biomater Sci, 2013, 1, 688-702.

17 D. E. Birk and P. Bruckner, Top Curr Chem, 2005, 247, 185-205.

18 S. Köster, H. M. Evans, J. Y. Wong and T. Pfohl, Biomacromolecules, 2008, 9, 199-207.

19 M.-M. Giraud-Guille, L. Besseau and R. Martin, J Biomech, 2003, 36, 1571-1579.

20 D. Eglin, K. Shaffran, J. Livage, T. Coradin and C. Perry, J. Mater. Chem., 2006, 16, 4220-4230.

21 C. Aimé, G. Mosser, G. Pembouong, L. Bouteiller and T. Coradin, Nanoscale, 2012, 4, 7127-7134.

22 A. O. Brightman, B. P. Rajwa, J. E. Sturgis, M. E. McCallister, J. P. Robinson and S. L. Voytik-Harbin, Biopolymers, 2000, 54, 222-234.

23 R. Pankov and A. Momchilova, Methods in Molecular Biology, Extracellular Matrix Protocols, 2009, 522, 261-274.

24 S.-J. Lin, J. Wu, Ruei, H.-Y. Tan, W. Lo, W.-C. Lin, T.-H. Young, C.-J. Hsu, J.-S. Chen, S.-H. Jee and C.-Y. Dong, Opt. Lett., 2005, 30, 22752277.

25 P. P. Provenzano, K. W. Eliceiri, J. M. Campbell, D. R. Inman, J. G. White and P. J. Keely, BMC Medicine, 2006, 4, 1-15.

26 N. D. Kirkpatrick, S. Andreou, J. B. Hoying and U. Utzinger, Am. J. Physiol., 2007, 292, H3198-H3206.

27 C. B. Raub, J. Unruh, V. Suresh, T. Krasieva, T. Lindmo, E. Gratton, B. J. Tromberg and S. C. George, Biophys. J., 2008, 94, $2361-2373$.

28 M. Strupler, M. Hernest, C. Fligny, J.-L. Martin, P.-L. Tharaux and M.-C. Schanne-Klein, J. Biomed. Optics, 2008, 13, 054041.

29 C. Bayan, J. M. Levitt, E. Miller, D. Kaplan and I. Georgakoudi, J. Appl. Phys., 2009, 105, 102042-102042-11.

30 W.-L. Chen, P.-S. Hu, A. Ghazaryan, S.-J. Chen, T.-H. Tsai and C.-Y. Dong, Computerized Medical Imaging and Graphics, 2012, 36, 519-526.

31 H. Altendorf, E. Decencière, D. Jeulin, P. De sa Peixoto, A. DenisetBesseau, E. Angelini, G. Mosser and M.-C. Schanne-Klein, J. Microsc., 2012, 247, 161-175.

32 E. Decencière, E. Tancrède-Bohin, P. Dokládal, S. Koudoro, A.-M. Pena and T. Baldeweck, Skin research and technology, 2013, 19, 115-124.

33 P. J. Campagnola, A. C. Millard, M. Terasaki, P. E. Hoppe, C. J. Malone and W. A. Mohler, Biophys. J., 2002, 81, 493-508.

34 T. Cedervall, I. Lynch, S. Lindman, T. Berggård, E. Thulin, H. Nilsson, K. A. Dawson and S. Linse, Proc Natl Acad Sci USA, 2007, 104, 20502055.

35 S. V. Patwardhan, F. S. Emami, R. J. Berry, S. E. Jones, R. R. Naik, O. Deschaume, H. Heinz and C. C. Perry, J Am Chem Soc, 2012, 134, 62446256.

36 S. Goy-Lopez, J. Juarez, M. Alatorre-Meda, E. Casals, V. F. Puntes, P. Taboada and M. Victor, Langmuir, 2012, 28, 9113-9126.

37 A. Deniset-Besseau, P. De Sa Peixoto, G. Mosser and M.-C. Schanne-
Klein, Opt. Express, 2010, 18, 1113-1121.

38 S. V. Plotnikov, A. Millard, P. Campagnola and W. Mohler, Biophys. J., 2006, 90, year.

39 A. Tuer, M. Akens, S. Krouglov, D. Sandkuijl, B. Wilson, C. Whyne and V. Barzda, Biophys. J., 2012, 103, 2093-2105.

40 J. Duboisset, A. Deniset-Besseau, E. Benichou, I. Russier-Antoine, N. Lascoux, C. Jonin, F. Hache, M.-C. Schanne-Klein and P.-F. Brevet, J. Phys. Chem. B, 2013, 117, 9877-9881.

41 S. Bancelin, C. Aimé, T. Coradin and M.-C. Schanne-Klein, Biomed. Opt. Express, 2012, 3, 1446-1454.

42 J. Serra, Image Analysis and Mathematical Morphology - Volume I, Academic Press, 1982.

43 P. Soille, Morphological image analysis, Springer-Verlag, 1999.

44 J. Serra, Journal of Mathematical Imaging and Vision, 1998, 9, 231-251.

45 A. van Blaaderen and A. Vrij, Langmuir, 1992, 8, 2921-2931. 